\title{
Cartoon + Texture Image Decomposition by the TV-L1 Model
}

\author{
Vincent Le Guen ${ }^{1}$ \\ ${ }^{1}$ TELECOM ParisTech, France (vincent.leguen@telecom-paristech.org) \\ Communicated by Jean-François Aujol Demo edited by Vincent Le Guen
}

\begin{abstract}
We consider the problem of decomposing an image into a cartoon part and a textural part. The geometric and smoothly-varying component, referred to as cartoon, is composed of object hues and boundaries. The texture is an oscillatory component capturing details and noise. Variational models form a general framework to obtain $u+v$ image decompositions, where cartoon and texture are forced into different functional spaces. The TV-L1 model consists in a $L^{1}$ data fidelity term and a Total Variation (TV) regularization term. The $L^{1}$ norm is particularly well suited for the cartoon+texture decomposition since it better preserves geometric features than the $L^{2}$ norm. The TV regularization has become famous in inverse problems because it enables to recover sharp variations. However, the nondifferentiability of TV makes the underlying problems challenging to solve. There exists a wide literature of variants and numerical attempts to solve these optimization problems. In this paper, we present an implementation of a primal dual algorithm proposed by Antonin Chambolle and Thomas Pock applied to this image decomposition problem with the TV-L1 model. A thorough experimental comparison is performed with a recent filter pair proposed in IPOL for the cartoon+texture decomposition.
\end{abstract}

\section{Source Code}

The source code and the online demonstration are accessible at the IPOL web part of this article $^{1}$.

Keywords: cartoon texture decomposition; total variation; image denoising

\footnotetext{
${ }^{1}$ https://doi.org/10.5201/ipol.2014.103
} 


\section{Introduction}

In what follows, we assume that a grayscale (respectively color) image can be represented by a function $f:(x, y) \in \Omega \longrightarrow \mathbb{R}$ (respectively $\mathbb{R}^{3}$ ) where $\Omega$ is an open subset of $\mathbb{R}^{2}$, typically a rectangle or a square. We assume that an image $f$ is defined on a continuous domain of $\mathbb{R}^{2}$. We get the continuous image by interpolating its corresponding digital image (defined on a discrete set of pixels). We are interested in decomposing $f$ into two components $f=u+v$ via a variational problem. The image $u$ represents the cartoon or geometric (piecewise-smooth) component of $f$ and $v$ the textured component that captures essentially the oscillatory patterns and the noise.

Following the formalism developed by Le and Vese [18], the general framework in order to decompose an image into $u+v$ is given by Meyer's models [21]

$$
\inf _{(u, v) \in X_{1} \times X_{2}}\left\{F_{1}(u)+\lambda F_{2}(v): f=u+v\right\}
$$

where $F_{1}, F_{2} \geq 0$ are functionals and $X_{1}, X_{2}$ are spaces of functions or distributions such that $X_{1}=\left\{u: F_{1}(u)<\infty\right\}$ and $X_{2}=\left\{v: F_{2}(v)<\infty\right\}$. The constant $\lambda>0$ is a tuning parameter. Many problems in imaging can be represented by the model (1), by an appropriate choice of $F_{1}$ and $F_{2}$. In our case, we are looking for two spaces $X_{1}$ and $X_{2}$, and two corresponding functionals $F_{1}$ and $F_{2}$, such that if $u$ is cartoon and $v$ is texture, we have $F_{1}(u)<<F_{2}(u)$ and $F_{1}(v)>>F_{2}(v)$. The texture components must be penalized by $F_{1}$ and not by $F_{2}$, and vice-versa for the cartoon components.

The algorithms proposed to solve this problem differ on the choices for $X_{1}, X_{2}$, and $F_{1}, F_{2}$. A good choice for $F_{1}$ is the total variation of $u$, that tends to involve constant regions and permits sharp edges, that are necessary for the cartoon part. It remains to discuss what space $X_{2}$ would model the oscillatory (textural) part. We refer to Table 1, taken from Buades et al. [4], and its legend, which presents the main models. This table extends the model classification outlined by Aujol et al. [3], and adopts the same terminology.

Let us recall the decomposition obtained by the Rudin, Osher, and Fatemi (ROF) total variation (TV) minimization model [28] for image denoising. Their functional is convex and therefore amenable to efficient minimization. The variational model is

$$
\inf _{(u, v) \in B V(\Omega) \times L^{2}(\Omega)}\left\{\int_{\Omega}|D u|+\lambda\|v\|_{L^{2}(\Omega)}^{2}, \quad f=u+v\right\}
$$

where

$$
\int_{\Omega}|D u|=\sup \left\{\int_{\Omega} u \operatorname{div} \vec{\phi} d x, \quad \vec{\phi} \in C_{0}^{1}\left(\Omega, \mathbb{R}^{2}\right), \quad\|\vec{\phi}\|_{\infty} \leq 1\right\}
$$

denotes the total variation of $u$ in $\Omega$, also denoted by $T V(u)$ or by $|u|_{B V(\Omega)}$. The component $u$ belongs to the space of functions of bounded variation $B V(\Omega)=\left\{u \in L^{1}(\Omega): \int_{\Omega}|D u|<\infty\right\}$.

The space BV penalizes oscillations (such as noise or texture), but allows for piecewise-smooth functions, made of homogeneous regions with smooth contrasted boundaries. Since almost all level lines (or isolines) of a $B V$ function have finite length, the $B V$ space is considered adequate to model images containing shapes.

The bibliography on algorithms minimizing the ROF functional and its multi-scale variants (Tadmor et al. [31], Strong et al. [30]) is rich (Aubert et al. [1], Vese et al. [33], Green [16], Osher et al. [25]). Hybrid models with wavelets are described in the papers by Malgouyres [20] and Lintner et al. [19].

We present in this paper an implementation of the TV-L1 model, where the $L^{2}$ norm is replaced by the $L^{1}$ norm. Non differentiable data like $L^{1}$ terms were inaugurated by Nikolova [23], and then studied by Chan and Esedoglu [9]. Many aspects of the TV-L1 model were covered in the literature: applications to outliers and impulse noise removal (Nikolova [24]), study of the TV-L1 model from a 


\begin{tabular}{|c|c|c|}
\hline Minimized energy or filters & Name & Reference \\
\hline $\begin{array}{c}\int|D u|^{2}+\mathcal{H}^{1}\left(J_{u}\right)+\int|v|^{2} \\
\int|D u|+\int|v|^{2} \\
\int|D u|+\int|v|\end{array}$ & $\begin{array}{c}S B V-L^{2} \\
T V-L^{2}(\mathrm{ROF}) \\
T V-L^{1}\end{array}$ & $\begin{aligned} & {[22] } \\
& {[28] } \\
\text { here }, & {[3,17,36] }\end{aligned}$ \\
\hline $\begin{array}{c}\int|D u|+\inf _{\vec{g} \in L^{\infty}, v=\operatorname{div} \vec{g}}\|\vec{g}\|_{L^{\infty}} \\
\int|D u|+\|v\|_{H^{-1}} \\
\int|D u|+\inf _{\vec{g} \in B M O, v=\operatorname{div} \vec{g}}\|\vec{g}\|_{L^{\infty}} \\
\int|D u|+\|v\|_{\dot{B}_{\infty, \infty}^{-1}}\end{array}$ & $\begin{array}{l}T V-\operatorname{div}\left(L^{\infty}\right) \\
\text { TV- } H^{-1} \\
\text { TV-div(BMO) } \\
\text { TV-Besov }\end{array}$ & $\begin{array}{c}{[21]} \\
{[26]} \\
{[21,18,13]} \\
{[21,14,2]}\end{array}$ \\
\hline $\begin{array}{c}\int|D u|+\int|K * v|^{2} \\
\int|D u|^{2}+\|v\|_{H^{-1}}^{2}\end{array}$ & $\begin{array}{l}\text { TV-Hilbert } \\
H^{1}-H^{-1}\end{array}$ & $\begin{array}{c}{[3]} \\
{[4] \text { and [29] }}\end{array}$ \\
\hline$u=w L_{\sigma} * f+(1-w) f$ & nonlinear filter pair & [4] and [5] \\
\hline
\end{tabular}

Table 1: Table of all $f=u+v=$ cartoon + texture models in approximate chronological order. These models are divided in five groups. The first group contains the classic $B V$ or $S B V+$ noise models. The second group starting with Meyer's model introduces a key new feature: the norm of the oscillatory part $v$ decreases when $v$ oscillates more. This is obtained by putting a norm on $v$ that is actually a norm on a primitive of $v$. The TV- $H^{-1}, \mathrm{TV}-\operatorname{div}(\mathrm{BMO})$ and TV-Besov models follow the same pattern. The third group simplifies the panorama by pointing out that the norm of a primitive of $v$ is much easier to compute by convolution with a filter $K$ (in fact the TV- $H^{-1}$ model also belongs to that group). The last row is the nonlinear filter proposed by Buades et al. [4], which takes the best of each worlds by using BV, but relying mainly on a previous pair of linear high-pass and low-pass filters. We shall compare here our implementation of the TV-L1 method to this fast filter, whose implementation was published in IPOL [5].

geometric point of view (Duval et al. [11], Yin et al. [37]), connection with mathematical morphology (Darbon [10]) and cartoon-texture decomposition (Aujol et al. [3], Haddad [17], Yin et al. [36], Chambolle and Pock [7]). The TV-L1 model is particularly well adapted to cartoon + texture decomposition. As noted by Duval et al. [11], TV-L1 kills small details and high curvatures and makes a thresholding on the ratio perimeter/area. The $v$ part contains objects with fine granulometry, whereas $u$ contains objects with coarse granulometry, which ensures informally that $T V(u) \ll\|u\|_{1}$ and $T V(v) \gg\|v\|_{1}$.

Let us review some existing methods for solving the TV-L1 problem. The first class of methods is based on smoothing the TV term :

$$
T V(u) \simeq \int_{\Omega} \sqrt{|D u|^{2}+\epsilon^{2}}
$$

We can cite the linearized gradient method proposed by Vogel and Oman [34]. It consists in solving the Euler-Lagrangian equation via a fixed-point equation, which implies to solve a linear system in each iteration. Another smoothing technique is the half-quadratic regularization approach [15], which is applied to the TV-L1 problem in the paper by Chan and Liang [8].

Another class of algorithms is based on the augmented Lagrangian method (ALM), which is applied to TV-L1 restoration by Zhang and Tai [35]. A variant is the alternating direction method (ADM) [32]. The main idea of ADM is to reformulate a TV problem as a linear equality con- 
strained problem where the objective function is separable and then minimize its augmented Lagrangian function. An online implementation of ADM is available at http://www.caam.rice.edu/ optimization/L1/RecPF/. A Matlab implementation of Chambolle's primal dual algorithm applied to image decomposition with a stationary noise assumption [12] is also available at : http: //www.math. univ-toulouse.fr/ weiss/PageCodes.html.

The rest of the paper is organized as follows: in Section 2, we present Chambolle's primal dual algorithm applied to the cartoon+texture decomposition problem and discuss the role of the parameters. In Section 3, we present experimental results and compare them with those obtained by Buades et al. [5].

\section{Chambolle-Pock Algorithm}

\subsection{The General Formulation in Finite Dimension}

Total variation minimization is a convex variational method that plays an important role in imaging as it allows for sharp discontinuities in the solution. However, it is known to be difficult to minimize due to the non-smoothness of the objective. First, we present the general formulation of the problem in finite dimension (we will therefore give a finite dimensional discretization of all operators in the next section).

Let $\mathcal{X}, \mathcal{Y}$ be two finite-dimensional real vector spaces equipped with an inner product $\langle\cdot, \cdot\rangle$ and norm $\|\cdot\|=\langle\cdot, \cdot\rangle^{\frac{1}{2}}$. Following the formalism of Chambolle and Pock [7], let $F: \mathcal{Y} \longrightarrow[0 ;+\infty[$ and $G: \mathcal{X} \longrightarrow\left[0 ;+\infty\left[\right.\right.$ be convex, lower-semicontinuous (l.s.c) functions. $F^{*}$ denotes the convex conjugate of the convex l.s.c. function $F$. We recall that the convex conjugate is defined by $F^{*}(y)=$ $\sup _{x \in \mathcal{Y}}\{\langle x, y\rangle-F(x)\}$.

Let $K: \mathcal{X} \longrightarrow Y$ be a linear operator with induced norm

$$
\|K\|=\sup \{\|K x\|: x \in \mathcal{X} \text { with }\|x\| \leq 1\} .
$$

The general problem we aim at solving, which will be referred as the primal problem, is

$$
\inf _{x \in \mathcal{X}} F(K x)+G(x) .
$$

Because of Fenchel Rockafellar's duality (see Rockafellar [27]), the corresponding dual problem is

$$
\sup _{y \in \mathcal{Y}}-G^{*}\left(-K^{T} y\right)-F^{*}(y)
$$

where $K^{T}$ denotes the transposed of $K$. There is no duality gap, therefore $(4)=(5)$.

By using the fact that $F(x)=\left(F^{*}\right)^{*}(x)=\sup _{y}\langle y, x\rangle-F^{*}(y)$, we can replace $F(K x)=$ $\sup _{y}\langle y, K x\rangle-F^{*}(y)$ in the primal problem, which yields the primal-dual problem

$$
\inf _{x \in \mathcal{X}} \sup _{y \in \mathcal{Y}} G(x)+\langle y, K x\rangle-F^{*}(y) .
$$

We recall that by definition the subdifferential of a convex and proper function $F: \mathcal{X} \rightarrow \mathbb{R}$ is the set-valued operator $\partial F: \mathcal{X} \rightarrow 2^{\mathcal{X}}$ whose value at $x \in \mathcal{X}$ is

$$
\partial F(x)=\{u \in \mathcal{X}: \forall z, F(z) \geq F(x)+\langle u, z-x\rangle\} .
$$

If $F$ is differentiable at $x$ then $\partial F(x)=\{\nabla F(x)\}$. A point $x^{*}$ is a minimizer of $F$ if and only if $0 \in \partial F\left(x^{*}\right)$. 
We assume that the problems (4), (5) and (6) have at least a solution $(\hat{x}, \hat{y}) \in \mathcal{X} \times \mathcal{Y}$. For a given $y$, we get from equation (6) that $0 \in\left(\partial G(\hat{x})+K^{T} y\right) \Longleftrightarrow-\left(K^{T} y\right) \in \partial G(\hat{x})$. For a given $x$, we get from equation (6) that $0 \in\left(-\partial F^{*}(\hat{y})+K x\right) \Longleftrightarrow K x \in \partial F^{*}(\hat{y})$. With the two previous relationships, a solution $(\hat{x}, \hat{y})$ satisfies:

$$
\begin{aligned}
K \hat{x} & \in \partial F^{*}(\hat{y}) \\
-\left(K^{*} \hat{y}\right) & \in \partial G(\hat{x}),
\end{aligned}
$$

where $\partial F^{*}$ and $\partial G$ are the subgradients of the convex function $F^{*}$ and $G$. We also suppose that $F$ and $G$ are "simple" functions in the sense that their proximal operators have a closed-form representation

$$
\operatorname{Prox}_{\gamma G}(x):=\underset{z}{\operatorname{argmin}} \frac{1}{2}\|x-z\|^{2}+\gamma G(z)=(\operatorname{Id}+\gamma \partial G)^{-1}(x),
$$

where $\gamma$ is a step-size and the last equality comes from the definition of the subdifferential.

\subsection{Discrete Setting}

We consider an image of size $M \times N:\left(u_{i, j}\right)_{1 \leq i \leq M, 1 \leq j \leq N}$. Let $\mathcal{X}=\mathbb{R}^{M N}$ be a finite dimensional vector space equipped with a standard scalar product

$$
\langle u, v\rangle_{\mathcal{X}}=\sum_{i, j} u_{i j} v_{i j}
$$

The gradient $\nabla u$ belongs to the vector field $\mathcal{Y}=\mathcal{X} \times \mathcal{X}$. In this section and in the rest, without risk of ambiguity we will continue writing $\nabla$ and div for the discrete operators associated with gradient and divergence. For discretization of $\nabla: \mathcal{X} \rightarrow \mathcal{Y}$, we use standard finite differences with Neumann boundary conditions

$$
(\nabla u)_{i, j}=\left(\begin{array}{c}
(\nabla u)_{i, j}^{1} \\
(\nabla u)_{i, j}^{2}
\end{array}\right)
$$

where

$$
\begin{aligned}
& (\nabla u)_{i, j}^{1}=\left\{\begin{array}{ll}
u_{i+1, j}-u_{i, j} & \text { if } i<M \\
0 & \text { if } i=M
\end{array},\right. \\
& (\nabla u)_{i, j}^{2}= \begin{cases}u_{i, j+1}-u_{i, j} & \text { if } j<N \\
0 & \text { if } j=N .\end{cases}
\end{aligned}
$$

We also define a scalar product in $\mathcal{Y}$, for all $p=\left(p^{1}, p^{2}\right)$ and $q=\left(q^{1}, q^{2}\right)$ by

$$
\langle p, q\rangle_{\mathcal{Y}}=\sum_{i, j} p_{i, j}^{1} q_{i, j}^{1}+p_{i, j}^{2} q_{i, j}^{2}
$$

We define the discrete divergence operator $\operatorname{div} p: \mathcal{Y} \longrightarrow \mathcal{X}$, as the adjoint of the gradient operator $\operatorname{div}=-\nabla^{*}$, defined through the identity

$$
\langle u, \operatorname{div}\rangle_{\mathcal{X}}=-\langle p, \nabla u\rangle_{\mathcal{Y}}
$$

It is easy to check that with this definition,

$$
(\operatorname{div}(\mathrm{p}))_{i, j}=\left\{\begin{array}{ll}
p_{i, j}^{1}-p_{i-1, j}^{1} & \text { if } 1<i<M \\
p_{i, j}^{1} & \text { if } i=1 \\
-p_{i-1, j}^{1} & \text { if } i=M
\end{array}+ \begin{cases}p_{i, j}^{2}-p_{i, j-1}^{2} & \text { if } 1<j<N \\
p_{i, j}^{2} & \text { if } j=1 \\
-p_{i, j-1}^{2} & \text { if } j=N .\end{cases}\right.
$$




\subsection{Application to the TV-L1 Model}

In the discrete setting, the TV-L1 model reads as:

$$
\min _{u} \lambda\|u-g\|_{1}+\|\nabla u\|_{1}
$$

where $g$ is the original image, and the solution of this problem $u^{*}$ will be the cartoon part. The discrete $L^{1}$ norm is defined by $\|u\|_{1}=\sum_{i, j}\left|u_{i j}\right|$ and for a vector field $\|\nabla u\|_{1}=\sum_{i, j} \sqrt{\left((\nabla u)_{i j}^{1}\right)^{2}+\left((\nabla u)_{i j}^{2}\right)^{2}}$.

We rewrite the problem in the standard form $\min _{u} F \circ K(u)+G(u)$ with $G(u)=\lambda\|u-g\|_{1}$, $F(u)=\|u\|_{1}$ and $K=\nabla$.

First, let us calculate the convex conjugate of the function $F(x)=\|x\|_{1}$, where $x \in \mathbb{R}^{d}$. By definition,

$$
\begin{aligned}
F^{*}(y) & =\sup _{x \in \mathbb{R}^{d}}\left\{\langle x, y\rangle_{\mathbb{R}^{d}}-|| x \|_{1}\right\} \\
& =\sup _{x \in \mathbb{R}^{d}}\left\{\sum_{i=1}^{d} x_{i} y_{i}-\left|x_{i}\right|\right\} .
\end{aligned}
$$

If there exists $k_{0}$ such as $y_{k_{0}}>1$ (respectively $y_{k_{0}}<-1$ ), then we have $F^{*}(y) \rightarrow+\infty$ when $x_{k_{0}} \rightarrow+\infty$ (respectively $x_{k_{0}} \rightarrow-\infty$ ). Hence, to have a finite objective, we have the condition $\left|y_{i}\right| \leq 1, \forall i \leq d$. Moreover, we have under this condition $\sum_{i=1}^{d} x_{i} y_{i}-\left|x_{i}\right| \leq \sum_{i=1}^{d} x_{i}-\left|x_{i}\right| \leq 0$, and the supremum is obtained when $x=0_{\mathbb{R}^{d}}$. We can thus write

$$
F^{*}(y)=\iota_{\|\cdot\|_{\infty} \leq 1}(y)
$$

where $\iota_{\mathcal{C}}$ denotes the indicator function of a convex set $\mathcal{C}$

$$
\iota_{\mathcal{C}}(x)= \begin{cases}0 & \text { if } x \in \mathcal{C} \\ \infty & \text { otherwise }\end{cases}
$$

F and $\mathrm{G}$ being convex, l.s.c. and simple functions, the conditions of Section 2.1 are met. Therefore we can derive the primal dual problem

$$
\begin{aligned}
\min _{u} \max _{p}\left\{G(u)+\langle\nabla u, p\rangle-F^{*}(p)\right\} & =\min _{u} \max _{p}\left\{\lambda\|u-g\|_{1}+\langle\nabla u, p\rangle-\iota_{\|\cdot\|_{\infty} \leq 1}(p)\right\} \\
& =\min _{u} \max _{p}\left\{\lambda\|u-g\|_{1}-\langle u, \operatorname{div} p\rangle-\iota_{\|\cdot\|_{\infty} \leq 1}(p)\right\},
\end{aligned}
$$

where we have used for the last equality that the adjoint operator of $\nabla$ is - div.

Chambolle's primal-dual algorithm solves the optimization problem with an alternate minimization scheme, by applying at each step the two proximal operators $\operatorname{Prox}_{\sigma F^{*}}$ and $\operatorname{Prox}_{\tau G}$. A direct calculation (Chambolle and Pock's paper [7], p.28) leads to

$$
\operatorname{Prox}_{\sigma F^{*}}(\tilde{p})=\left(\operatorname{Id}+\sigma \partial F^{*}\right)^{-1}(\tilde{p})=\frac{\tilde{p}_{i j}}{\max \left(1,\left|\tilde{p}_{i j}\right|\right)},
$$

and

$$
\operatorname{Prox}_{\tau G}(\tilde{u})=(\operatorname{Id}+\tau \partial G)^{-1}(\tilde{u})= \begin{cases}\tilde{u}_{i j}-\tau \lambda & \text { if } \tilde{u}_{i j}-g_{i j}>\tau \lambda \\ \tilde{u}_{i j}+\tau \lambda & \text { if } \tilde{u}_{i j}-g_{i j}<-\tau \lambda \\ g_{i j} & \text { if }\left|\tilde{u}_{i j}-g_{i j}\right| \leq \tau \lambda\end{cases}
$$


Each step uses a fixed-point iteration. If $G$ a "simple" function (namely we know its proximal operator), then we have the fixed point equation

$$
\begin{aligned}
x^{*} \in \underset{x}{\operatorname{argmin}} F(x)+G(x) & \Longleftrightarrow 0 \in \partial F\left(x^{*}\right)+\partial G\left(x^{*}\right) \\
& \Longleftrightarrow\left(x^{*}-\gamma \partial F\left(x^{*}\right)\right) \in x^{*}+\gamma \partial G\left(x^{*}\right) \\
& \Longleftrightarrow x^{*}=\operatorname{Prox}_{\gamma G}\left(x^{*}-\gamma \partial F\left(x^{*}\right)\right) .
\end{aligned}
$$

We therefore have the iteration

$$
x_{k+1}=\operatorname{Prox}_{\gamma G}\left(x_{k}-\gamma \partial F\left(x_{k}\right)\right) .
$$

The primal-dual algorithm (Chambolle and Pock [7]) is summarized below. We alternatively minimize over the two variables $p$ and $u$ with a fixed-point iteration. We refer to their paper [7] for results of convergence of this algorithm.

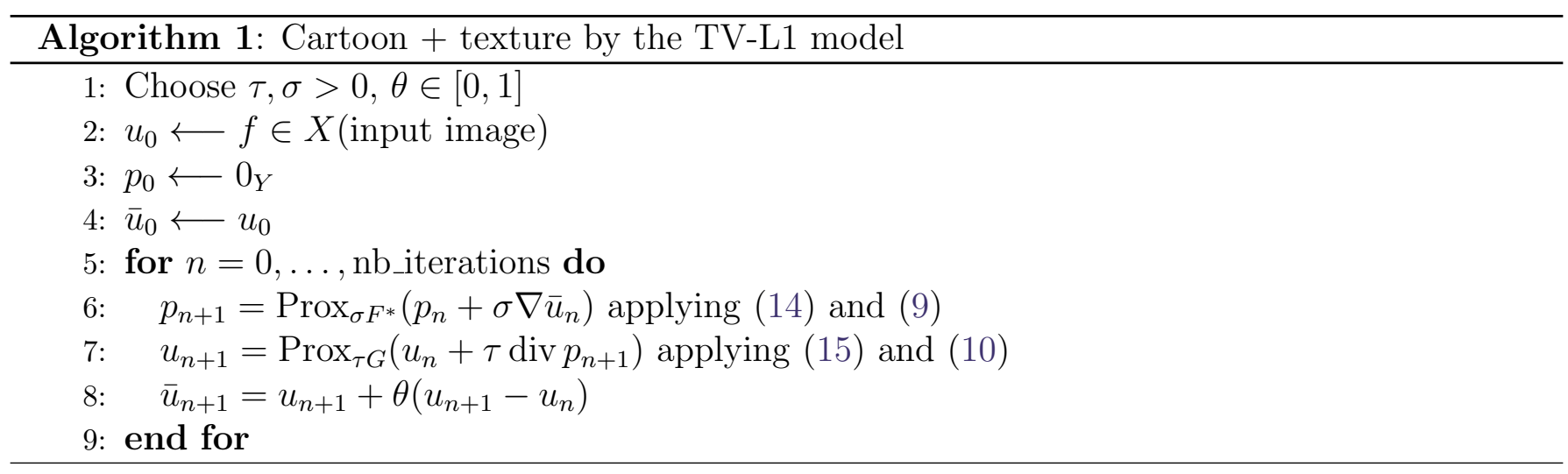

Color images can be handled by processing each channel independently with Algorithm 1.

\subsection{The Parameters}

The parameters $\sigma$ and $\tau$ are the steps in the iterative scheme. The parameter $\theta$ defines the extrapolation step. Chambolle and Pock [7] give a proof of convergence under the conditions $\theta=1$ and $\tau \sigma L^{2} \leq 1$, where $L^{2}=\|\nabla\|^{2} \leq 8$ (for a proof of the last inequality, see Chambolle's paper [6]). In the source code, we use the scheme $\theta=1$ and the parameters $\tau=\sigma=0.35$ to satisfy the constraint. The parameter $\lambda$ controls the tradeoff between the fidelity term (closeness to the original image) and the regularization term. The lower $\lambda$, the smoother the image. All other parameters being fixed, we found experimentally that the range $[0.01 ; 2]$ gives very convincing results. So $\lambda=0.01$ corresponds to a very strong cartoon regularization and $\lambda=2$ to almost no regularization.

In Figure 1, we display the results for several values of $\lambda$. For example, we remark that the tiles on the tablecloth progressively vanish when $\lambda$ decreases (i.e. when there is more regularization): this is exactly what we wanted.

To adjust the number of iterations, we use a stopping criterion with the threshold $10^{-3}$ on the decreasing normalized energy

$$
\frac{1}{N M}\left(\lambda\|u-g\|_{1}+\|\nabla u\|_{1}\right) .
$$

It enables a greater number of iterations for small $\lambda$. For example, for the fingerprint image (Figure 3), 90 iterations are needed for $\lambda=1$ and 220 iterations for $\lambda=0.1$. 


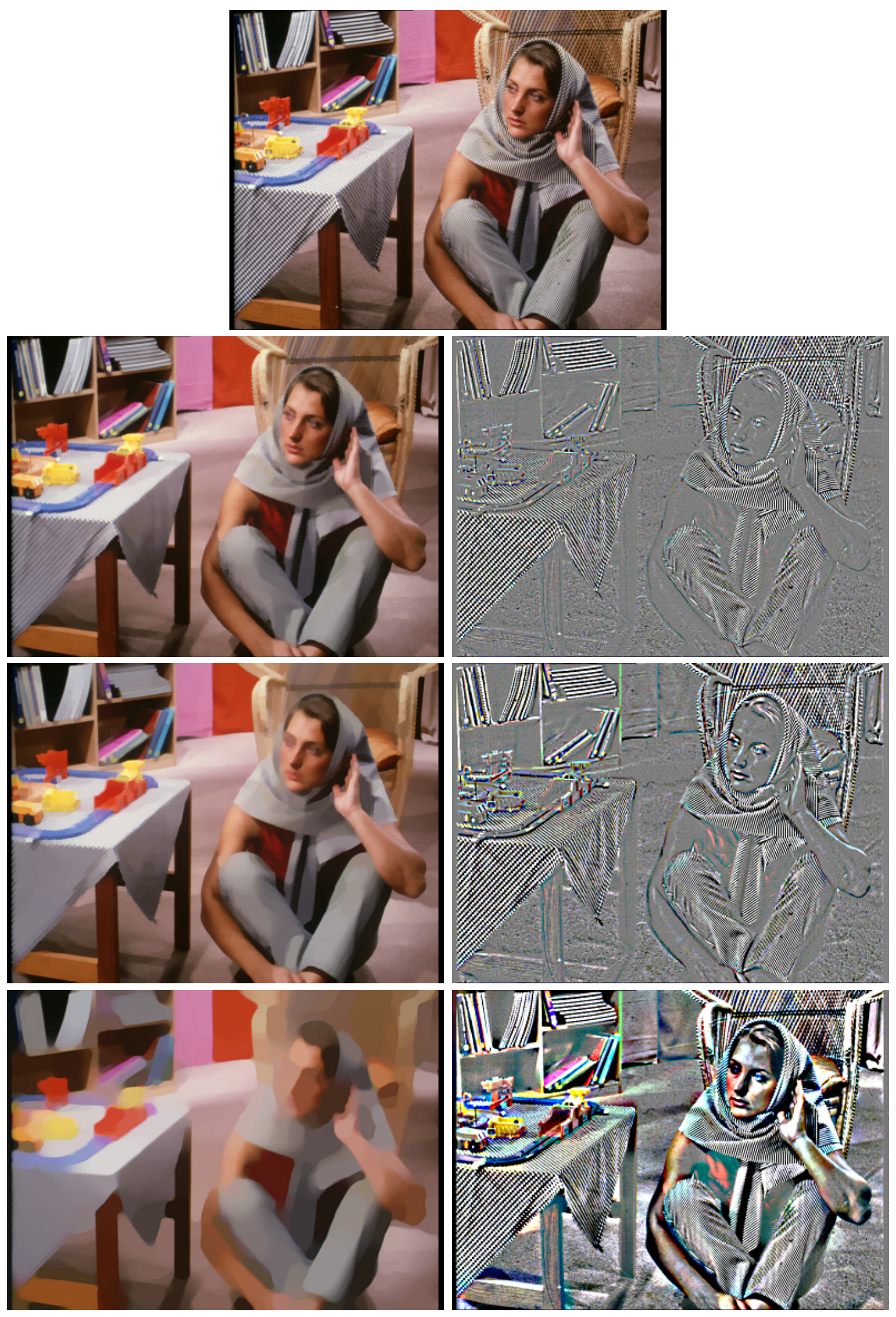

Figure 1: Influence of $\lambda$. First row: original image. Second row: $\lambda=0.7$. Third row: $\lambda=0.4$. Fourth row: $\lambda=0.1$. 


\section{Experimental Results}

We present in this section examples of decomposition and compare them with those obtained by Buades et al. [4]. We will refer to this algorithm as the nonlinear low pass-high pass filter (NLHF). And we will refer to the TV-L1 decomposition algorithm by TV-L1. For the definition of the scale parameter, we refer to their paper [4].

\subsection{A Brief Description of NLHF}

The nonlinear low pass-high pass filter (NLHF) for cartoon+texture decomposition proceeds as follows: for each image point $x$, a decision is made of whether it belongs to the cartoon part or to the textural part. This decision is made by computing a local total variation of the image around the point

$$
\operatorname{LTV}_{\sigma}(x)(u):=G_{\sigma} *|\nabla f|(u),
$$

where $G_{\sigma}$ is a Gaussian kernel with standard deviation sigma. This is compared to the local total variation after a low pass filter has been applied to the image, resulting in an indicator of the relative reduction rate of LTV.

Edge points in an image tend to have a slowly varying local total variation when the image is convolved by a low pass filter, whereas there is a strong decay for textural points. According to this decision for each pixel, the cartoon part keeps a weighted average of the filtered value and the original value. The texture part simply is the difference between the original image and its cartoon part.

\subsection{Methodology}

In order to compare the decomposition results obtained by the NLHF and the TV-L1 algorithms, the BV-norms of the cartoon parts were equated. We recall that in the discrete setting, the BV-norm of an image $u$ is defined by

$$
\|u\|_{B V}=\|\nabla u\|_{1}=\sum_{i, j} \sqrt{\left((\nabla u)_{i j}^{1}\right)^{2}+\left((\nabla u)_{i j}^{2}\right)^{2}} .
$$

More precisely, the NLHF algorithm was run with the same value of $\sigma$ as in Buades et al.'s IPOL implementation [5]. Then the chosen $\lambda$ for the TV-L1 algorithm was the one giving approximately the same BV norm for the cartoon part. This choice was performed by dichotomy. In the NLHF algorithm, the higher $\sigma$, the smoother the image and thus the smaller the BV norm of the cartoon part. So, the BV norm of the cartoon is an decreasing function of $\sigma$. On the contrary, for the TV-L1 algorithm, the BV norm of the cartoon is an increasing function of $\lambda$.

\subsection{Results}

In the dolphin image (Figure 2), we see almost no difference in the cartoon part since the original image is already cartoon-like. In the fingerprint image (Figure 3), the finger edges are better recovered with the TV-L1 algorithm. We see the same phenomenon with the cactus image (Figure 4), with the texture square (Figure 8) and the noisy square (Figure 9). So TV-L1 performs better than NLHF near edges.

The choice of $\lambda$ depends on the application. For the dolphin image (Figure 2) which is already an almost perfect cartoon, a small regularization is needed and thus we can choose a large value for $\lambda$. On the contrary for the fingerprint (Figure 3), a large regularization is needed to remove the whole texture and thus we choose a small $\lambda$ (for example 0.1). 

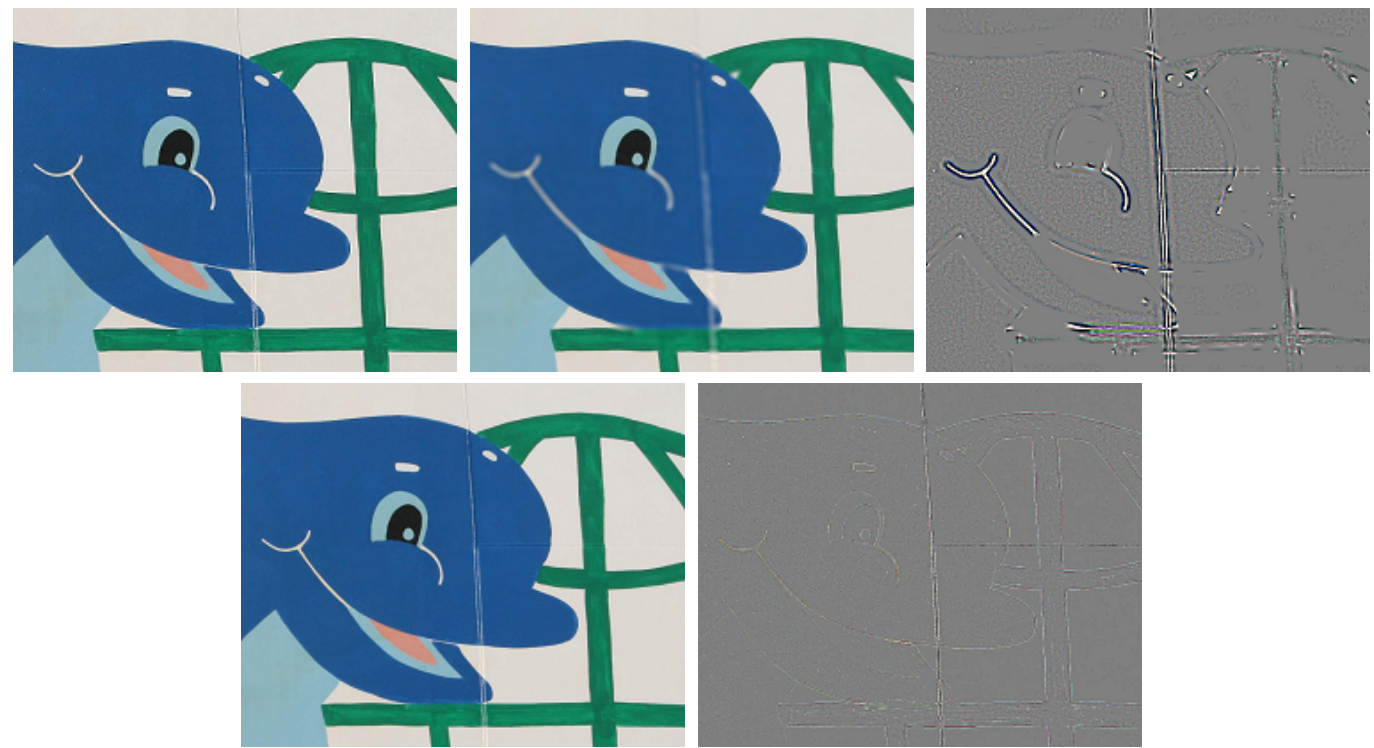

Figure 2: Dolphin. First row: original image, cartoon and texture with NLHF $(\sigma=4)$. Second row: TV-L1 algorithm $(\lambda=1.6)$.
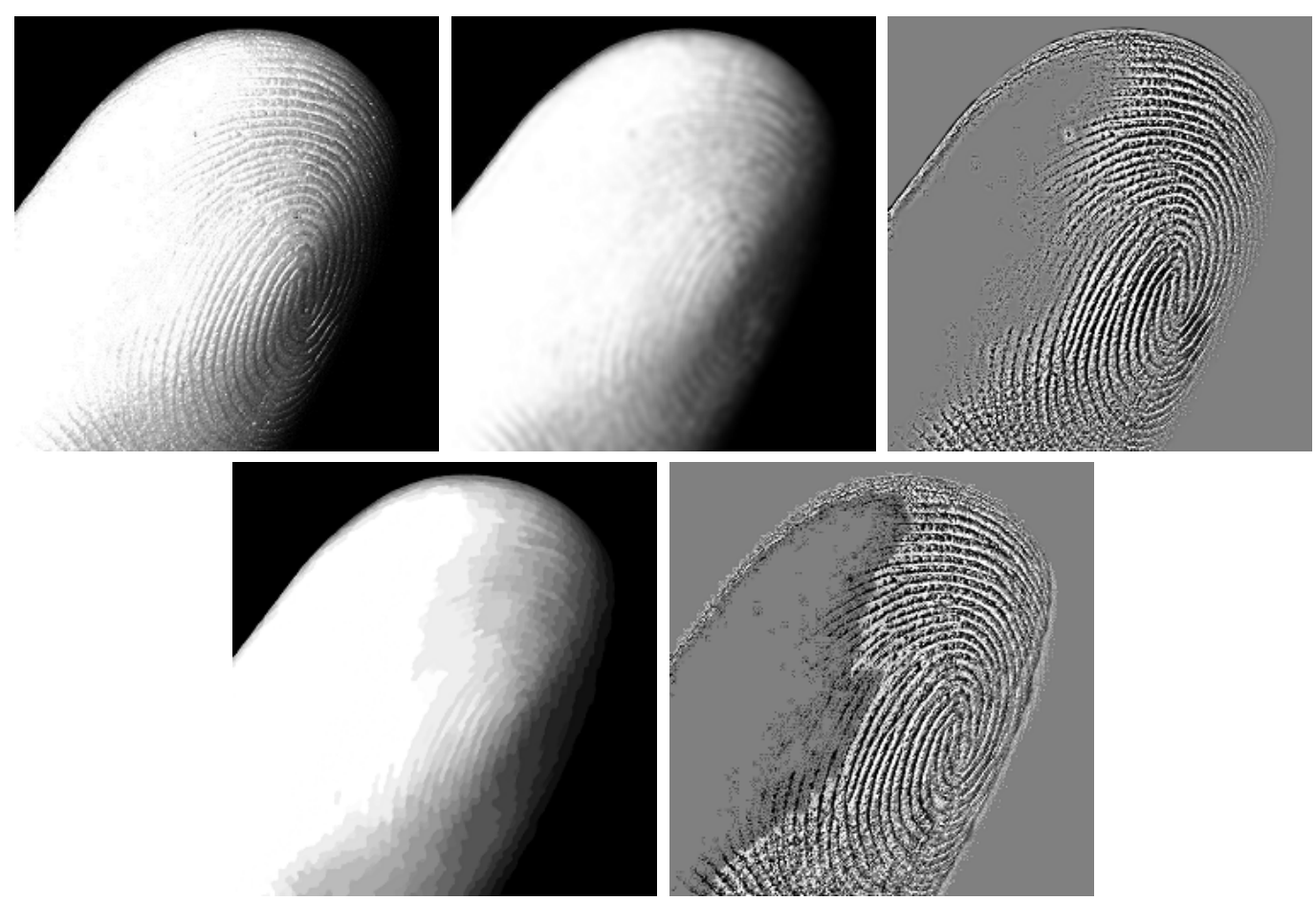

Figure 3: Fingerprint. First row: original image, cartoon and texture with NLHF $(\sigma=5)$. Second row: TV-L1 algorithm $(\lambda=0.5)$. Notice the obviously better separation of cartoon and texture with TV-L1. 

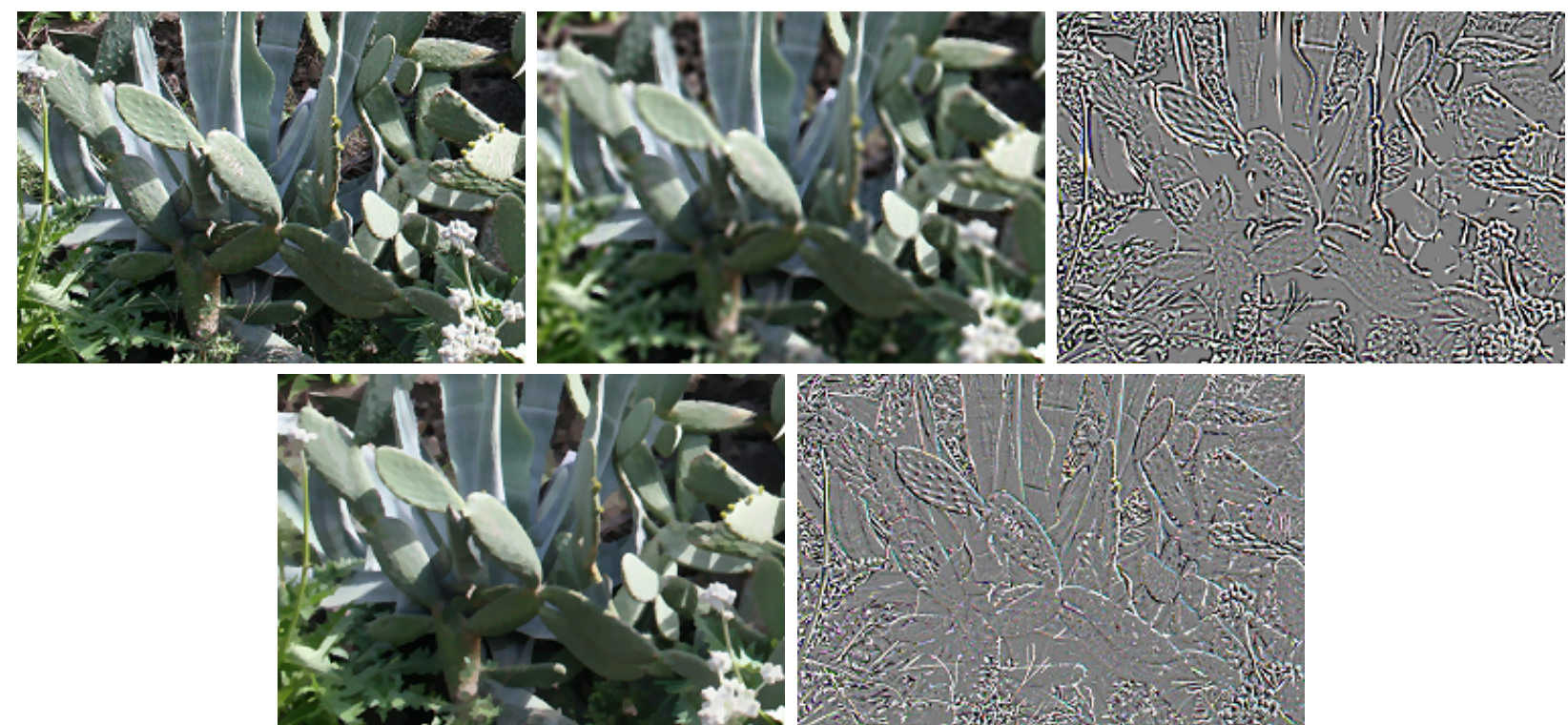

Figure 4: Cactus. First row: original image, cartoon and texture with NLHF $(\sigma=5)$. Second row: TV-L1 algorithm $(\lambda=0.6)$.
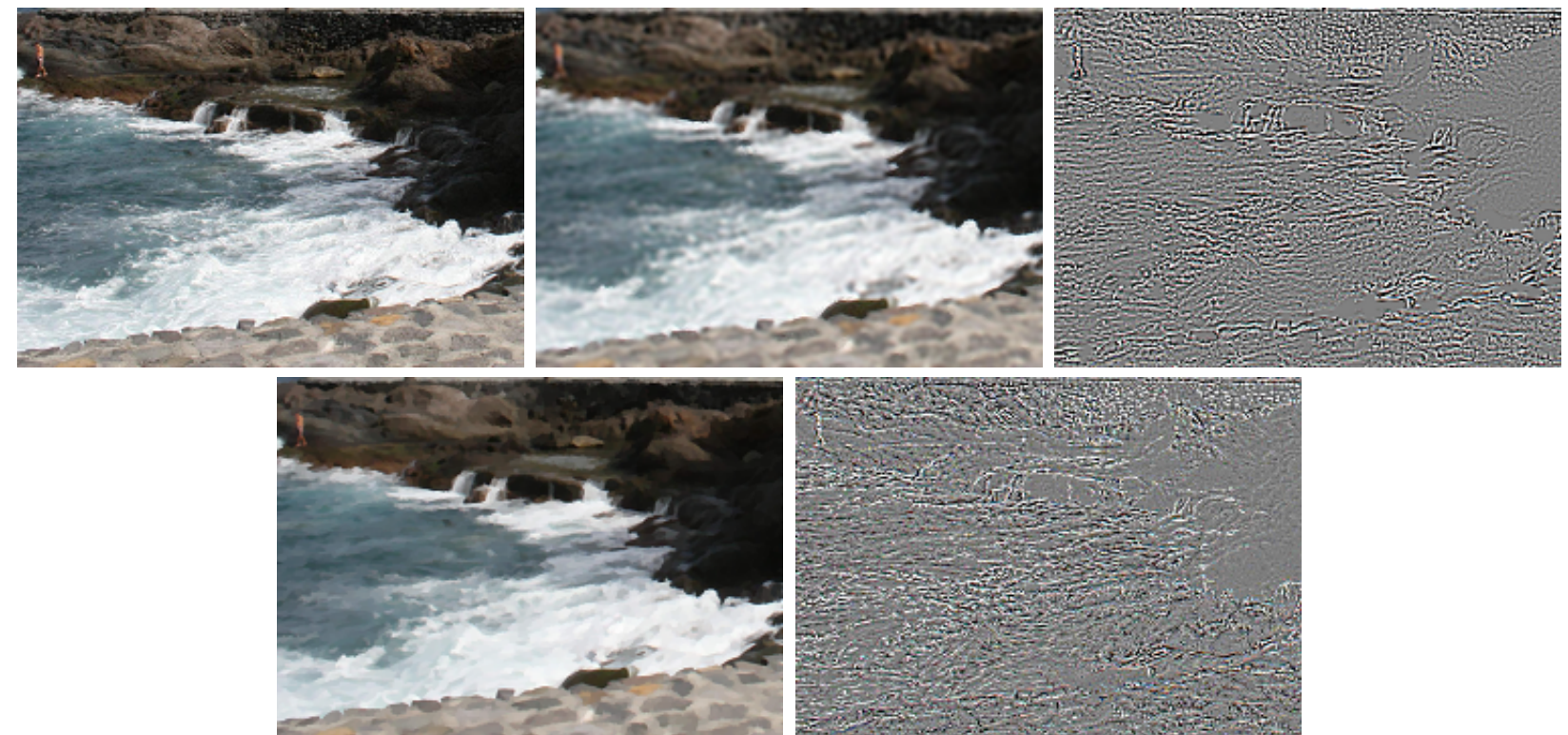

Figure 5: Waves. First row: original image, cartoon and texture with NLHF $(\sigma=4)$. Second row: TV-L1 algorithm $(\lambda=0.7)$. 

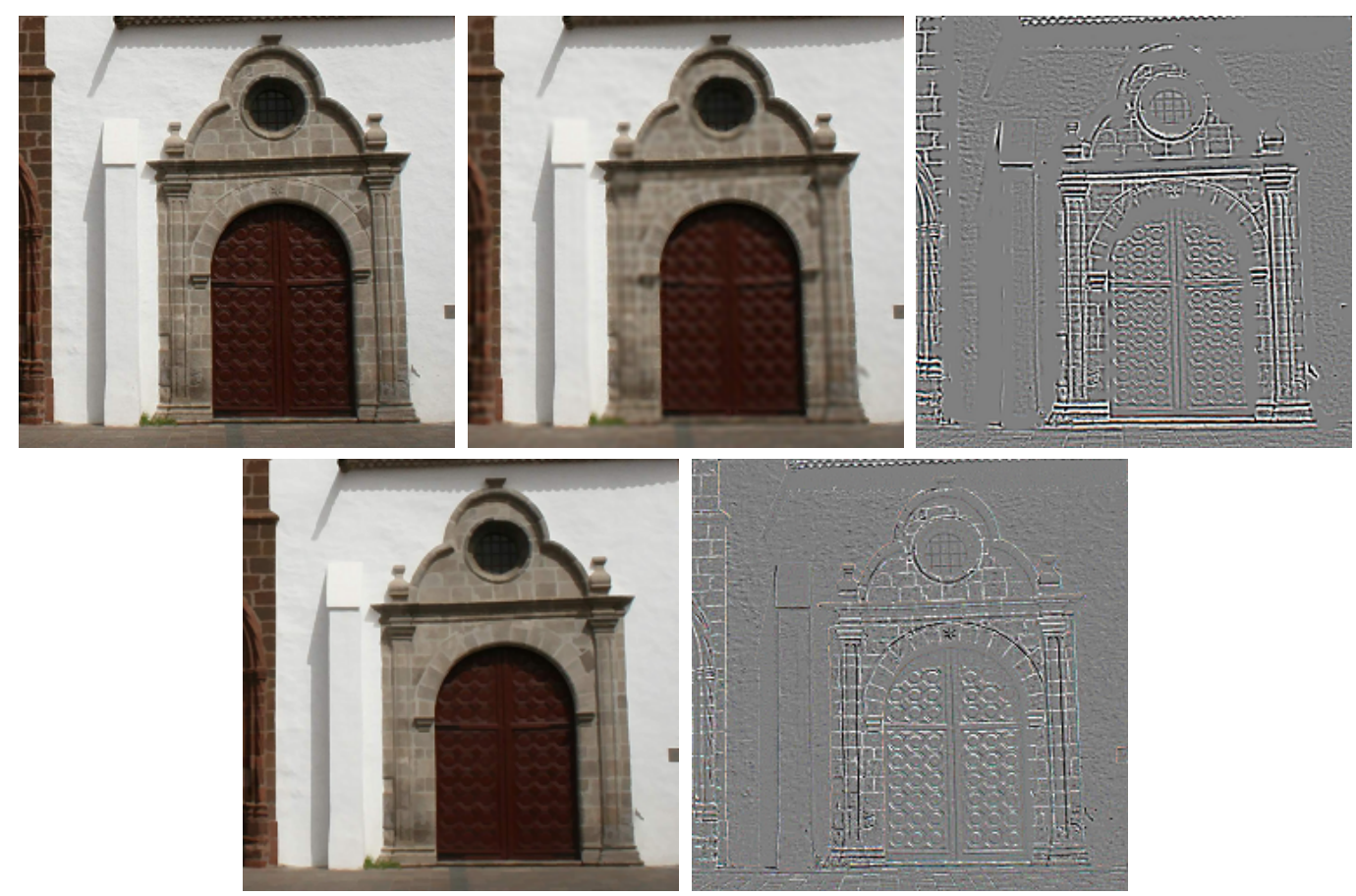

Figure 6: Church. First row: original image, cartoon and texture with NLHF $(\sigma=5)$. Second row: TV-L1 algorithm $(\lambda=0.7)$. The cartoon-texture separation is clearly sharper with TV-L1.
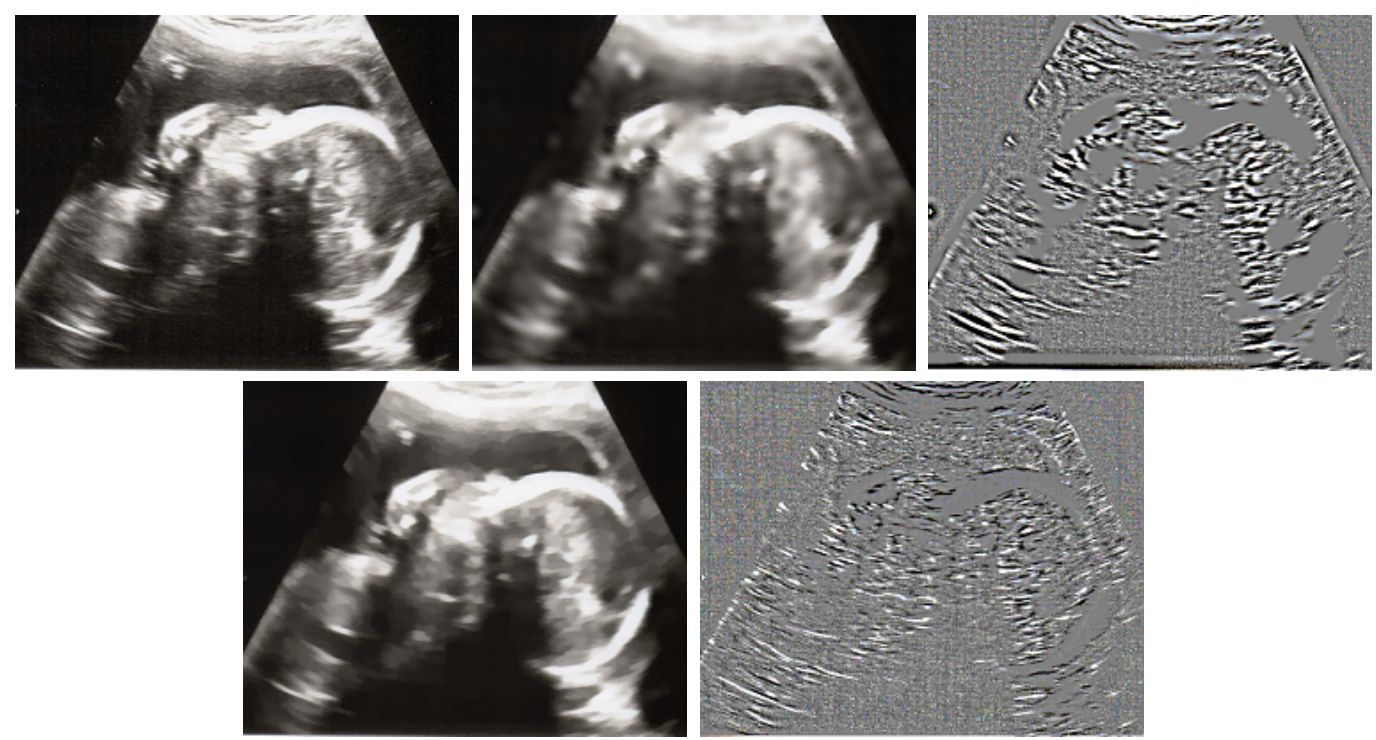

Figure 7: Ultrasound image. First row: original image, cartoon and texture with NLHF $(\sigma=5)$. Second row: TV-L1 algorithm $(\lambda=0.7)$. 

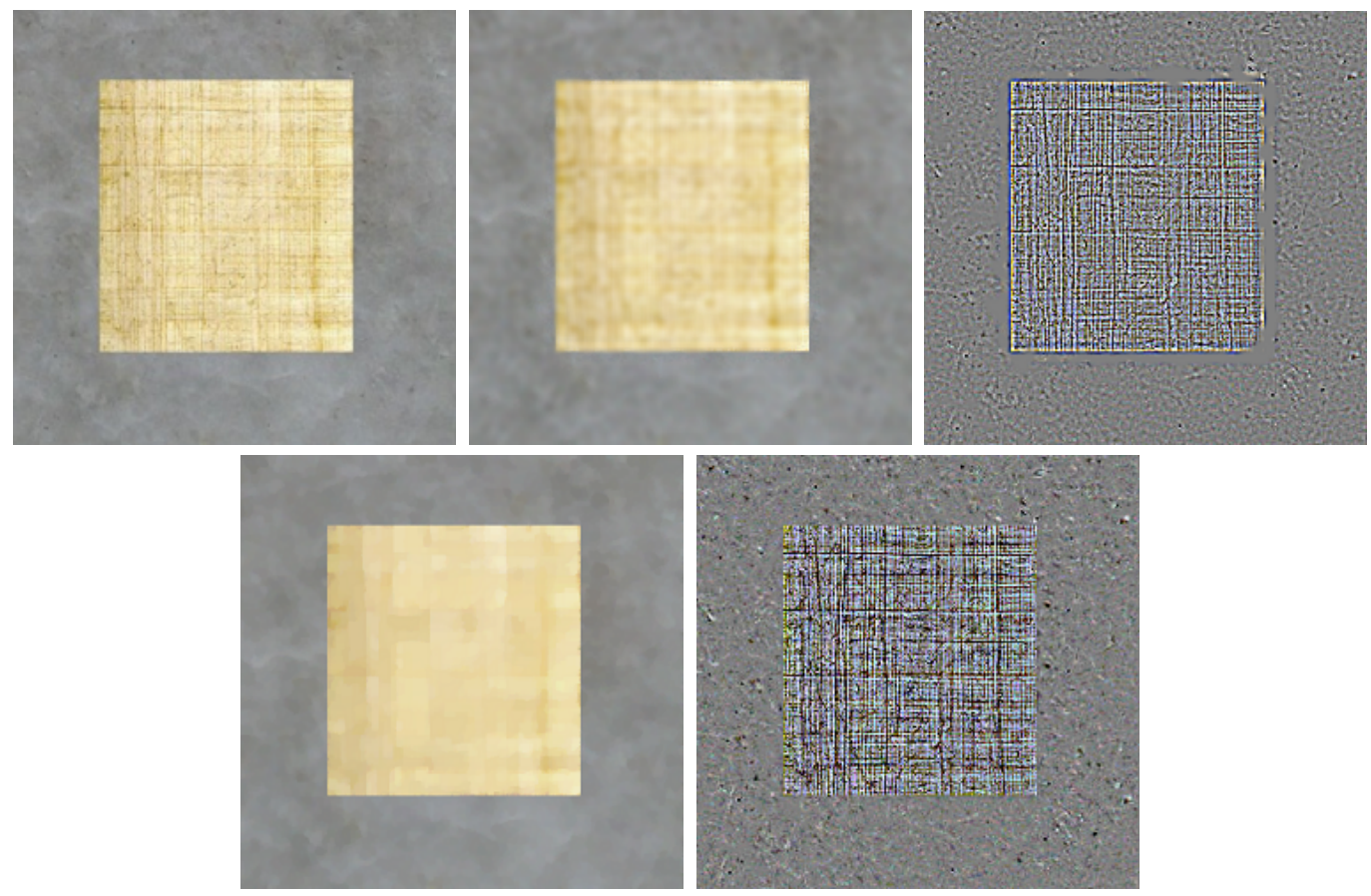

Figure 8: Texture square. First row: original image, cartoon and texture with NLHF $(\sigma=3)$. Second row: TV-L1 algorithm $(\lambda=0.8)$. Remark that the cartoon part is sharper with TV-L1 and the texture separation better.
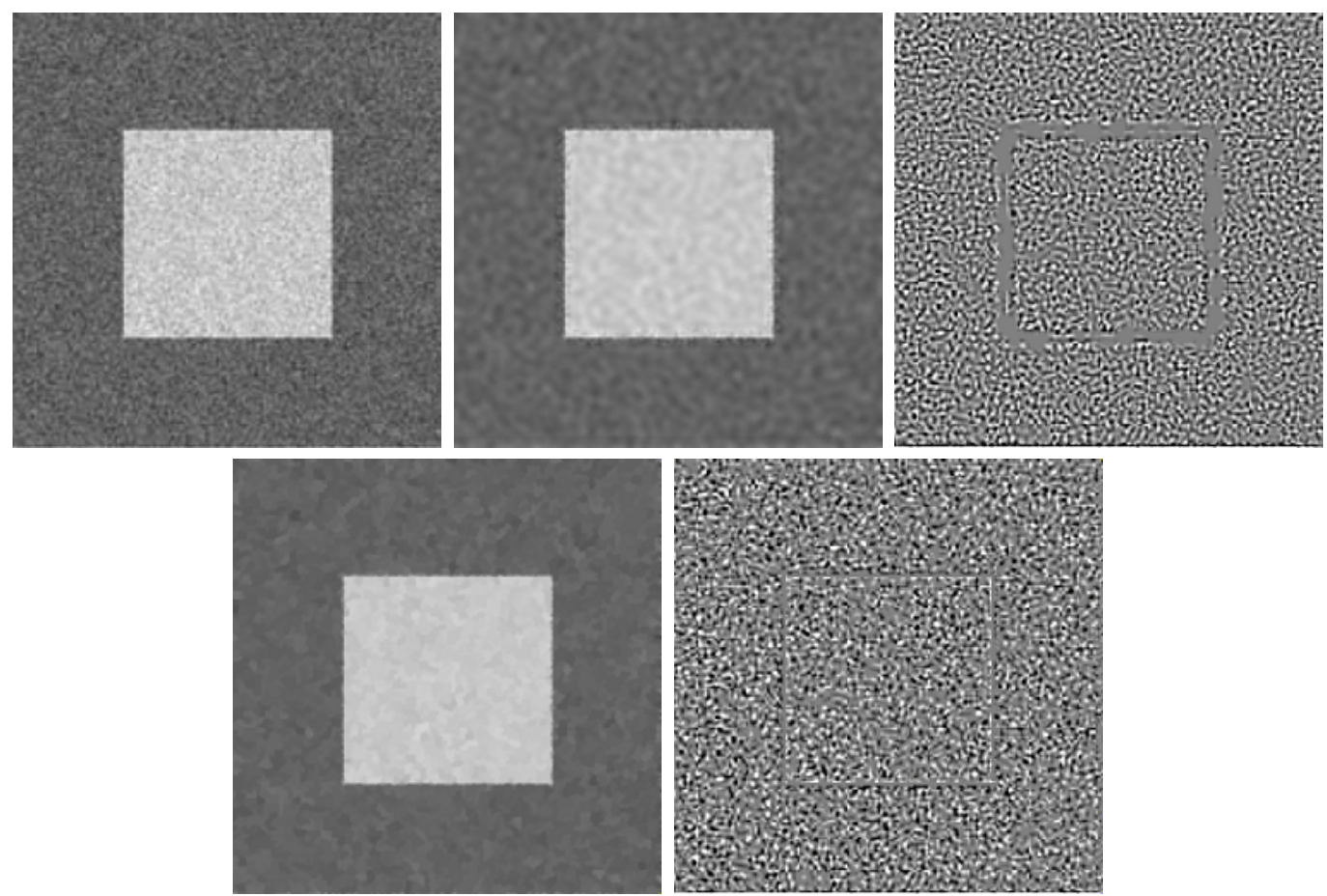

Figure 9: Noisy square. First row: original image, cartoon and texture with NLHF $(\sigma=3)$. Second row: TV-L1 algorithm $(\lambda=0.8)$. We remark that the TV-L1 gives significantly better results near edges. 


\section{Image Credits}

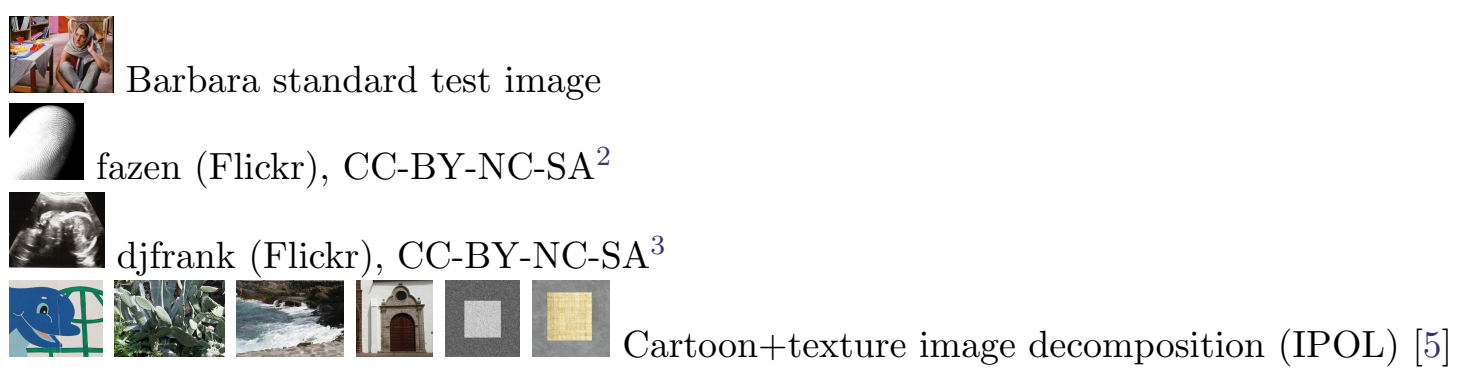

\section{References}

[1] G. Aubert And L. Vese, A variational method in image recovery, SIAM Journal on Numerical Analysis, 34 (1997), pp. 1948-1979. http://dx.doi.org/10.1137/S003614299529230X.

[2] J.-F. Aujol And A. Chambolle, Dual norms and image decomposition models, International Journal of Computer Vision, 63 (2005), pp. 85-104. http://dx.doi.org/10.1007/ s11263-005-4948-3.

[3] J.-F. Aujol, G. Gilboa, T. Chan, And S. Osher, Structure-texture image decompositionmodeling, algorithms, and parameter selection, International Journal of Computer Vision, 67 (2006), pp. 111-136. http://dx.doi.org/10.1007/s11263-006-4331-z.

[4] A. Buades, T.M. Le, J.-M. Morel, And L. Vese, Fast cartoon+texture image filters, IEEE Transactions on Image Processing, 19 (2010), pp. 1978-1986. http://dx.doi.org/10.1109/ TIP. 2010.2046605.

[5] — Cartoon+Texture Image Decomposition, Image Processing On Line, 2011 (2011). http: //dx.doi.org/10.5201/ipol.2011.blmv_ct.

[6] A. Chambolle, An algorithm for total variation minimization and applications, Journal of Mathematical Imaging and Vision, 20 (2004), pp. 89-97. http://dx.doi.org/10.1023/B: JMIV.0000011325.36760.1e.

[7] A. Chambolle And T. Pock, A first-order primal-dual algorithm for convex problems with applications to imaging, Journal of Mathematical Imaging and Vision, 40 (2011), pp. 120-145. http://dx.doi.org/10.1007/s10851-010-0251-1.

[8] R. H. Chan And H.-X. Liang, A fast and efficient half-quadratic algorithm for TV-L1 image restoration, The Chinese University of Hong Kong, (2011), pp. 1-14.

[9] T. Chan And S. Esedoglu, Aspects of total variation regularized $L^{1}$ function approximation, SIAM Journal on Applied Mathematics, 65 (2005), pp. 1817-1837. http://dx.doi.org/10. $1137 / 040604297$.

[10] J. DARBon, Total variation minimization with $L^{1}$ data fidelity as a contrast invariant filter, in Proceedings of the 4th International Symposium on Image and Signal Processing and Analysis (ISPA), September 2005, pp. 221-226. http://dx.doi.org/10.1109/ISPA.2005.195413.

\footnotetext{
${ }^{2}$ https://www.flickr.com/photos/fazen/3778408/

${ }^{3}$ https://www.flickr.com/photos/djfrank/5256374935/
} 
[11] V. Duval, J.-F. Aujol, And Y. Gousseau, The TV-L1 model: a geometric point of view, Multiscale Modeling \& Simulation, 8 (2009), pp. 154-189. http://dx.doi.org/10.1137/ 090757083.

[12] J. Fehrenbach, P. Weiss, And C. Lorenzo, Variational algorithms to remove stationary noise: applications to microscopy imaging, IEEE Transactions on Image Processing, 21 (2012), pp. 4420-4430. http://dx.doi.org/10.1109/TIP.2012.2206037.

[13] J. Garnett, P. Jones, T. Le, And L. Vese, Modeling oscillatory components with the homogeneous spaces BMO- $\alpha$ and $W$ - $\alpha, p$. UCLA CAM Report 07-21, 2007.

[14] J. Garnett, T. Le, Y. Meyer, And L. Vese, Image decompositions using bounded variation and generalized homogeneous Besov spaces, Applied and Computational Harmonic Analysis, 23 (2007), pp. 25-56. http://dx.doi.org/10.1016/j.acha.2007.01.005.

[15] D. GEMAn And C. YANG, Nonlinear image recovery with half-quadratic regularization, IEEE Transactions on Image Processing, 4 (1995), pp. 932-946. http://dx.doi.org/10.1109/83. 392335.

[16] M. Green, Statistics of images, the TV algorithm of Rudin-Osher-Fatemi for image denoising and an improved denoising algorithm. UCLA CAM Report 02-55, 2002.

[17] A Haddad, Texture separation $B V-G$ and $B V-L^{1}$ models, Multiscale Modeling \& Simulation, 6 (2007), pp. 273-286. http://dx.doi.org/10.1137/060670249.

[18] T. LE AND L. VESE, Image decomposition using total variation and div(BMO), Multiscale Modeling \& Simulation, 4 (2005), pp. 390-423. http://dx.doi.org/10.1137/040610052.

[19] S. Lintner And F. Malgouyres, Solving a variational image restoration model which involves $L^{\infty}$ constraints, Inverse Problems, 20 (2004), p. 815. http://dx.doi.org/10.1088/ 0266-5611/20/3/010.

[20] F. Malgouyres, Mathematical analysis of a model which combines total variation and wavelet for image restoration, Journal of Information Processing, 2 (2002), pp. 1-10.

[21] Y. MEYER, Oscillating patterns in image processing and nonlinear evolution equations: the fifteenth dean Jacqueline B. Lewis memorial lectures, vol. 22, AMS Bookstore, 2001.

[22] D. Mumford And J. Shah, Optimal approximations by piecewise smooth functions and associated variational problems, Communications on Pure and Applied Mathematics, 42 (1989), pp. 577-685. http://dx.doi.org/10.1002/cpa.3160420503.

[23] M. NikOLOva, Minimizers of cost-functions involving nonsmooth data-fidelity terms. application to the processing of outliers, SIAM Journal on Numerical Analysis, 40 (2002), pp. 965-994. http://dx.doi.org/10.1137/S0036142901389165.

[24] _ A variational approach to remove outliers and impulse noise, Journal of Mathematical Imaging and Vision, 20 (2004), pp. 99-120. http://dx.doi.org/10.1023/B: JMIV. $0000011326.88682 . e 5$

[25] S. Osher, M. Burger, D. Goldfarb, J. Xu, and W. Yin, An iterative regularization method for total variation-based image restoration, Multiscale Modeling \& Simulation, 4 (2005), pp. 460-489. http://dx.doi.org/10.1137/040605412. 
[26] S. Osher, A. SolÉ, AND L. VESE, Image decomposition and restoration using total variation minimization and the $H^{1}$ norm, Multiscale Modeling \& Simulation, 1 (2003), pp. 349-370. http://dx.doi.org/10.1137/S1540345902416247.

[27] R. Rockafellar, Convex analysis, vol. 28, Princeton University Press, $1997 . \quad$ ISBN 9780691015866.

[28] L. Rudin, S. Osher, And E. FAtemi, Nonlinear total variation based noise removal algorithms, Physica D: Nonlinear Phenomena, 60 (1992), pp. 259-268. http://dx.doi.org/10. 1016/0167-2789(92) 90242-F.

[29] J. Shen, Piecewise $H^{-1}+H^{0}+H^{1}$ images and the Mumford-Shah-Sobolev model for segmented image decomposition, Applied Mathematics Research Express, 2005 (2005), pp. 143-167.

[30] D. Strong And T. Chan, Edge-preserving and scale-dependent properties of total variation regularization, Inverse Problems, 19 (2003), p. S165. http://dx.doi.org/10.1088/0266-5611/ 19/6/059.

[31] E. Tadmor, S. Nezzar, And L. Vese, A multiscale image representation using hierarchical $\left(B V, L^{2}\right)$ decompositions, Multiscale Modeling \& Simulation, 2 (2004), pp. 554-579. http: //dx.doi.org/10.1137/030600448.

[32] M. TAO, J. YAng, AND B. He, Alternating direction algorithms for total variation deconvolution in image reconstruction. TR0918, Department of Mathematics, Nanjing University, 2009 .

[33] L. Vese, A Study in the BV Space of a Denoising-Deblurring Variational Problem, Applied Mathematics and Optimization, 44 (2001), pp. 131-161. http://dx.doi.org/10.1007/ s00245-001-0017-7.

[34] C. R. Vogel And M. E. Oman, Iterative methods for total variation denoising, SIAM Journal on Scientific Computing, 17 (1996), pp. 227-238. http://dx.doi.org/10.1137/0917016.

[35] C. Wu, J. Zhang, AND X.-C. TAI, Augmented lagrangian method for total variation restoration with non-quadratic fidelity, Inverse problems and imaging, 5 (2011), pp. 237-261. http://dx.doi.org/10.3934/ipi.2011.5.237.

[36] W. Yin, D. Goldfarb, And S. Osher, A comparison of three total variation based texture extraction models, Journal of Visual Communication and Image Representation, 18 (2007), pp. 240-252. http://dx.doi.org/10.1016/j.jvcir.2007.01.004.

[37] _ The total variation regularized $L^{1}$ model for multiscale decomposition, Multiscale Modeling \& Simulation, 6 (2007), pp. 190-211. http://dx.doi.org/10.1137/060663027. 